\title{
Genome-wide expression profiles of subchondral bone in osteoarthritis
}

\author{
Ching-Heng Chou ${ }^{1,2,3}$, Chia-Chun Wu ${ }^{4}$, I-Wen Song ${ }^{1,2,5}$, Hui-Ping Chuang ${ }^{6}$, Liang-Suei Lu ${ }^{1,2}$, Jen-Huei Chang ${ }^{4}$, \\ San-Yuan Kuo ${ }^{4}$, Chian-Her Lee ${ }^{7,8}$, Jer-Yuarn Wu ${ }^{1,2,6}$, Yuan-Tsong Chen ${ }^{1,9}$, Virginia Byers Kraus ${ }^{10,3}$ \\ and Ming Ta Michael Lee ${ }^{1,2,11,12^{*}}$
}

\begin{abstract}
Introduction: The aim of this study was to evaluate, for the first time, the differences in gene expression profiles of normal and osteoarthritic (OA) subchondral bone in human subjects.

Methods: Following histological assessment of the integrity of overlying cartilage and the severity of bone abnormality by micro-computed tomography, we isolated total RNA from regions of interest from human OA $(n=20)$ and non-OA $(n=5)$ knee lateral tibial (LT) and medial tibial (MT) plateaus. A whole-genome profiling study was performed on an Agilent microarray platform and analyzed using Agilent GeneSpring GX11.5. Confirmatory quantitative reverse-transcription polymerase chain reaction (qRT-PCR) analysis was performed on samples from 9 OA individuals to confirm differential expression of 85 genes identified by microarray. Ingenuity Pathway Analysis (IPA) was used to investigate canonical pathways and immunohistochemical staining was performed to validate protein expression levels in samples.

Results: A total of 972 differentially expressed genes were identified (fold change $\geq \pm 2, P \leq 0.05$ ) between LT (minimal degeneration) and MT (significant degeneration) regions from OA samples; these data implicated 279 canonical pathways in IPA. The qRT-PCR data strongly confirmed the accuracy of microarray results $\left(R^{2}=0.58\right.$, $P<0.0001)$. Novel pathways were identified in this study including Periostin (POSTN) and Leptin (LEP), which are implicated in bone remodeling by osteoblasts.

Conclusions: To the best of our knowledge, this study represents the most comprehensive direct assessment to date of gene expression profiling in OA subchondral bone. This study provides insights that could contribute to the development of new biomarkers and therapeutic strategies for OA.
\end{abstract}

\section{Introduction}

Osteoarthritis $(\mathrm{OA})$ is the most prevalent musculoskeletal disorder and the most common form of arthritis among older individuals in most countries worldwide; OA constitutes a large economic burden due to the associated costs of medical care and lost wages [1,2]. Although degeneration of cartilage is the major characteristic of OA, the disease also involves the entire joint organ, including structural modifications of underlying subchondral bone, pathological changes of the meniscus and synovitis $[3,4]$.

\footnotetext{
* Correspondence: mikelee@src.riken.jp

'Institute of Biomedical Sciences, Academia Sinica, 128, Sec. 2, Academia Road, Nankang District, Taipei 11529, Taiwan

${ }^{2}$ National Center for Genome Medicine, Institute of Biomedical Sciences, Academia Sinica, 128, Sec. 2, Academia Road, Nankang District, Taipei 11529, Taiwan

Full list of author information is available at the end of the article
}

Maintenance of normal joint structure and function relies on load adaptation of the cartilage and bone. Disruption of the physiological relationship between these tissues contributes to the development of OA pathology [5].

Increasing evidence indicates that the subchondral bone, consisting of the subchondral plate and subchondral spongiosa, plays a major role in the initiation and progression of OA [6]. Magnetic resonance imagingbased visualization of the whole knee structure demonstrates that increased tibial subchondral bone volume is associated with severity of knee OA [7]. Kraus and colleagues demonstrated that subchondral bone texture can be used as a biomarker to predict progression of knee OA [8]. A study comparing two guinea pig strains indicates that an increasing rate of knee subchondral bone remodeling is associated with the process of cartilage 
deterioration [9]. Moreover, human bone cell culture studies have shown that factors released from bone cells might affect cartilage metabolism [10,11]. These studies provide insights into a temporal relationship between subchondral structural changes and alterations in articular cartilage during the development of $\mathrm{OA}$; they also underscore the importance of elucidating the molecular changes in human subchondral bone to improve our understanding of the pathogenesis of OA.

Whole-genome microarray is a common technology for studying the behavior of many genes simultaneously. All of the gene expression microarray profiling studies in OA so far have been performed on human articular cartilage, meniscus or synovium; however, none have been performed on human subchondral bone tissue directly. Only one study has reported the gene expression profiles of subchondral bone in an early OA experimental mouse model [12]. A few studies have evaluated the gene expression profiles of distal trabecular bone from human OA $[13,14]$, but this site is remote from subchondral bone rather than a reflection of alterations locally in the OA joint. This paucity of subchondral bone microarray studies is most probably due to the difficulties associated with isolation of high-quality RNA from subchondral bone. As described in our previous study [15], we have developed a method of precisely harvesting specific regions of subchondral bone tissue and for subsequently isolating high-quality RNA from these specimens. Our methodology has made it possible to perform microarray analyses of human subchondral bone samples.

Our goal in this study was to evaluate the association of subchondral bone gene expression with bone histomorphometry at sites of intact articular cartilage and osteoarthritic lesioned cartilage. To our knowledge, this is the first study to successfully perform microarray analyses of human knee subchondral bone in OA and nonOA samples, thereby providing clues to the pathogenic mechanisms of OA that could inform development of new diagnostic markers and therapeutic targets.

\section{Methods}

\section{Human knee joint tissues}

Human osteoarthritic tibial plateaus with medial compartment dominant OA and macroscopically normal lateral compartments were obtained during total knee joint replacement surgery from knee OA patients. The diagnosis of OA was based on the criteria for knee OA of the American College of Rheumatology [16]. Normal tibial plateaus were obtained at autopsy or within 8 hours after amputation surgery from donors with nonarthritic knee joints.

To ensure consistency of sampling of prespecified regions of interest, the anatomic orientation was indicated on the freshly isolated specimens by marker pen and then all specimens were stored immediately in liquid nitrogen. Methods for precisely obtaining specimens of a particular type from specific regions of interest of the joint have been described previously [15]. The tissues were manipulated at all times under liquid nitrogen to prevent RNA degradation. Regions of interest were harvested for histological analysis of the articular cartilage, and subchondral bone structural parameters were determined by micro-computed tomography; regions immediately adjacent were harvested for RNA isolation and microarray analysis. For microarrays, RNA was isolated from subchondral bone from four regions: the outer and inner lateral tibial (LT) plateau and the inner and central medial tibial (MT) plateau (Additional file 1). The gene expression intensities changed little within the LT plateau (between inner and outer LT regions) and within the MT plateau (inner and central MT regions) (Additional file 2); we therefore based the analyses in the current study on a comparison of gene expression from subchondral bone of 20 outer LT plateaus and 20 MT plateaus from knee OA specimens. These 40 samples were derived from 30 individual knee OA specimens, yielding 10 paired LT and MT samples and 10 unpaired LT and MT samples. The $40 \mathrm{OA}$ samples chosen for microarray analysis represented a subset from our previous study [15], and were chosen to have LT OARSI scores $\leq 8$ and MT OARSI scores $\geq 18$. In addition, five LT and five MT paired subchondral bone samples from non-OA donors were used as controls for the microarray analysis. The characteristics of the samples used for microarray analysis are summarized in Table 1 and Additional file 3.

The study was approved by the institutional review board of Academia Sinica, Tri-Service General Hospital and Taipei Medical University Hospital, and written informed consent was obtained from all of the participants.

\section{Subchondral bone tissue harvest and RNA isolation}

All tissues were sectioned to generate osteochondral slabs 2 to $2.5 \mathrm{~mm}$ thick from regions of interest for microcomputed tomography scanning and histological analysis (Figure 1); regions immediately adjacent were isolated for subsequent grinding and RNA isolation using a custommade workstation as described previously [15]. Approximately $100 \mathrm{mg}$ powdered subchondral bone from the tibial plateau was obtained for RNA extraction. Five milliliters of Trizol (Invitrogen, Carlsbad, CA, USA) were added to $100 \mathrm{mg}$ (dry weight) ground tissue powder and mixed by vortexing until homogeneous. A total of $1 \mathrm{ml}$ chloroform was added to the homogenized sample and centrifuged at $12,000 \times g$ for 15 minutes at $4^{\circ} \mathrm{C}$ to achieve phase separation. The clear aqueous phase was transferred to a fresh tube for a second phase separation with phenol/ chloroform/isoamyl alcohol (25:24:1; Sigma-Aldrich, St. Louis, MO, USA), followed by RNA precipitation with pure 
Table 1 Characteristics of the samples used for microarray analysis

\begin{tabular}{lcccc}
\hline & \multicolumn{2}{c}{ Osteoarthritis } & \multicolumn{2}{c}{ Nonosteoarthritis } \\
\cline { 2 - 4 } Characteristic & LT & MT & $3.00 \pm 1.85$ & MT \\
\hline OARSI & $4.7 \pm 1.34$ & $20.8 \pm 1.62^{*}$ & $24.41 \pm 3.71$ & $3.75 \pm 1.67$ \\
BV/TV $\left(\mathrm{mm}^{3}\right)$ & $21.32 \pm 7.47$ & $65.39 \pm 12.61^{*}$ & $0.99 \pm 0.21$ & $0.68 \pm 0.73$ \\
SMl & $1.56 \pm 0.34$ & $-1.69 \pm 1.70^{*}$ & $0.19 \pm 0.01$ & $0.26 \pm 0.08$ \\
Tb.Th (mm) & $0.18 \pm 0.04$ & $0.36 \pm 0.09^{*}$ & $1.28 \pm 0.21$ & $1.36 \pm 0.09$ \\
Tb.N (1/mm) & $1.16 \pm 0.27$ & $1.83 \pm 0.33^{*}$ & $0.56 \pm 0.09$ & $0.49 \pm 0.03$ \\
Tb.Sp (mm) & $0.51 \pm 0.12$ & $0.25 \pm 0.09^{*}$ & $38.40 \pm 13.45$ & $38.40 \pm 13.45$ \\
Age (years) & $71.10 \pm 9.55$ & $69.65 \pm 9.55$ & 36 & 32 \\
Male sex (\%) & 33 & 34 & 5 & 5 \\
Number & 20 & 20 & &
\end{tabular}

Values presented as mean \pm standard deviation. BV/TV, percent bone volume; LT, lateral tibial; MT, medial tibial; OARSI, histological scoring system to grade severity of osteoarthritis; SMI, structure model index; Tb.N, trabecular number; Tb.Sp, trabecular separation; Tb.Th, trabecular thickness. ${ }^{*} P<0.001$ considered significant comparing MT osteoarthritis versus LT osteoarthritis. $P$ values were determined by two-tailed unpaired Student's $t$ test.

isopropanol. After washing with 70\% ethanol, the air-dried pellet was dissolved in RNase-free water and genomic DNA was removed by DNase digestion (Qiagen, Hilden, Germany) according to the manufacturer's instructions. To further improve RNA quality, one-half volume of $7.5 \mathrm{M} \mathrm{LiCl}$ (Invitrogen) was added and incubated at $-20^{\circ} \mathrm{C}$ for at least 30 minutes followed by RNA precipitation by centrifugation at $12,000 \times g$ for 15 minutes. After $70 \%$ ethanol washing, the RNA was resuspended in RNase-free water. The RNA concentration and purity were determined by Nano-Drop (NanoDrop Technologies, Wilmington, DE, USA). The RNA integrity number and the $28 \mathrm{~s} / 18 \mathrm{~s}$ ratio were estimated using RNA 6000
Nano Assays on an Agilent 2100 Bioanalyzer (Agilent Technologies, Santa Clara, CA, USA) according to the manufacturer's instructions.

\section{Microarray analysis}

Four hundred nanograms of total RNA per sample were used for one round of cRNA synthesis and amplification. Cyanine 3-labeled cRNA was then purified and hybridized to Agilent whole human genome $44 \mathrm{k}$ microarray chips (Agilent Technologies). All procedures were carried out according to the manufacturer's instructions (Agilent Technologies, Taipei, Taiwan). The array signal
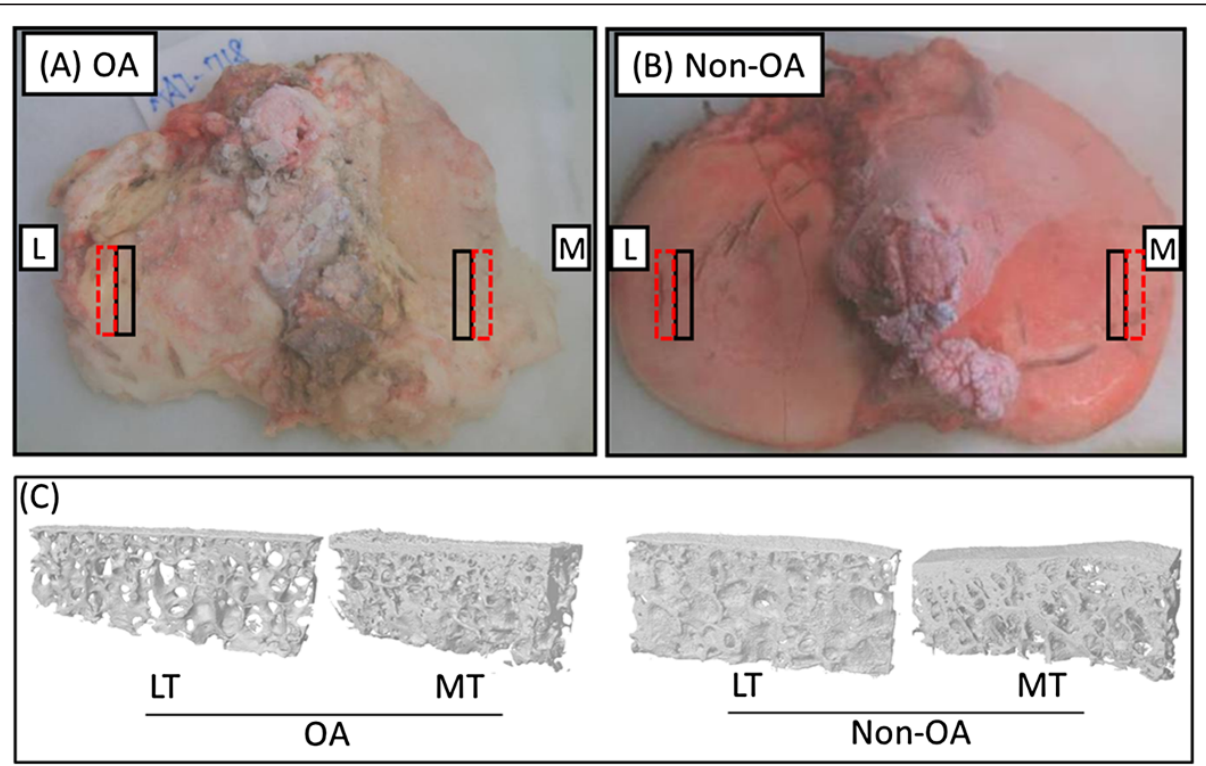

Figure 1 Sites of micro-computed tomography analysis and regions chosen for RNA isolation. (A) Human osteoarthritic knee tibial plateau (osteoarthritis (OA)) and (B) normal knee tibial plateau (non-OA). Red dashed boxes, regions chosen for RNA isolation; black boxes, regions chosen for micro-computed tomography and histological analysis at the two regions of interest. (C) Representative three-dimensional images of subchondral bone from the lateral tibial (LT) and medial tibial (MT) plateau from OA and non-OA specimens. 
intensities were further analyzed by the Agilent GeneSpring GX software (version 11.5; Agilent Technologies).

Gene expression values of all datasets were normalized using quantile normalization; probes with low signal intensities were excluded by setting the filter above 32 . The normalized values were log transformed. Significant differentially expressed genes between lateral and medial samples in OA patients were identified using an adjusted Student's $t$ test $(P<0.05)$ corrected for multiple comparisons with the Benjamini-Hochberg test. The results were used to run principal component analysis to uncover the trends among OA and normal samples. Differentially expressed genes between medial and lateral regions among groups were identified by a threshold of $\geq 2$ fold-change and $P \leq 0.05$. The hierarchical clustering method, with Euclidean distance and centroid linkage, was further applied to cluster the differences in gene expression levels between samples. Lists of differentially expressed genes were imported into ingenuity pathway analysis (IPA; Ingenuity Systems, Redwood City, CA, USA) to identify functional annotations and biological interactions from the many known relationships between proteins, genes and diseases. The biological interaction scores were defined by the IPA statistical algorithm based on its knowledge base, and the adjusted $P$ value was calculated by Fisher's exact test and corrected for multiple comparisons with the BenjaminiHochberg test. All microarray raw data are available through the GEO database [GEO:GSE51588].

\section{Quantitative reverse-transcription polymerase chain reaction validation}

To validate results from microarray analysis, we performed reverse-transcription polymerase chain reaction (qRT-PCR) for 85 genes (plus GAPDH) on nine additional OA paired LT and MT subchondral bone specimens; eight of these additional OA knee joint specimens have been described previously [15], as well as the qRT-PCR results for some of the genes combined with data yielded from one additional knee OA specimen. Forty-three of these genes were identified for verification on the basis of significant fold-change $(\geq 2)$ comparing LT and MT microarray results. The remaining 42 genes were identified on the basis of their potential involvement in $\mathrm{OA}$ as described previously [15]. The expression of these 42 genes was previously analyzed for seven regions of interest from eight OA cartilage and subchondral bone specimens.

In the current study, we analyzed two regions of interest (LT and MT subchondral bone corresponding to outer LT and center MT of the prior work) in nine specimens (the eight former specimens and an additional OA specimen with available LT and MT regions). The qRT-PCR was performed using the Taqman high-density microfluidic cards (Invitrogen, Carlsbad, CA) according to the manufacturer's instructions. RNA from each region was converted into cDNA using Superscript III reverse transcriptase (Invitrogen, Carlsbad, CA). The qRT-PCR reactions were performed on the ABI Prism 7900HT Sequence Detection system and the fluorescent signal intensity was analyzed by Sequence Detector v2.3 software (Applied Biosystems, Foster City, California). The levels of cDNA among samples were normalized to the expression of GAPDH and analyzed with the $\Delta \Delta \mathrm{Ct}$ relative quantification method to identify significant variation in gene expression comparing LT and MT regions in OA samples. Two-tailed unpaired Student $t$ tests were performed to evaluate for statistically significant differences in gene expression levels between regions.

\section{Immunohistochemistry staining}

Approximately $2 \mathrm{~mm}$ diameter sections from the tibial plateau were fixed in $4 \%$ paraformaldehyde (SigmaAldrich) overnight and decalcified in $10 \%$ ethylenediamine- $N, N, N^{\prime}, N^{\prime}$-tetraacetic acid, disodium salt, dehydrate (Sigma-Aldrich) for 2 weeks. After decalcification, the sections were embedded in paraffin and $10 \mu \mathrm{m}$ sections were prepared. The embedded tissue sections were then deparaffinized, hydrated, and the endogenous peroxidase activity was quenched in $0.3 \% \mathrm{H}_{2} \mathrm{O}_{2}$-methanol for 30 minutes. After incubating in $0.5 \%$ trypsin $/ 1 \% \mathrm{CaCl}_{2}$ at $37^{\circ} \mathrm{C}$ for 30 minutes and blocking with diluted goat serum at $25^{\circ} \mathrm{C}$ for 1 hour, the sections were incubated with anti-human PSOTN (dilution 1:300) antibodies (ab14041; Abcam, Cambridge, MA, USA), and LEP (dilution 1:1,000) antibodies (ab16627; Abcam) or nonimmune goat serum at $4{ }^{\circ} \mathrm{C}$ overnight and reacted with biotinylated secondary antibody for 30 minutes. Signal amplification and staining were performed using Vectastain ABC kits (Vector Laboratory, Burlingame, CA, USA) according to the manufacturer's protocol and counterstained with hematoxylin solution Gill no. 3 (Sigma-Aldrich).

\section{Results}

\section{Analysis of differential gene expression}

For the microarray analysis, 972 differentially expressed genes with $\geq 2$ fold-changes between OA MT and OA LT regions were identified; 420 of these genes were upregulated and 552 were downregulated in OA MT compared with OA LT (Additional file 4). In total, 640 genes were differentially expressed with greater than twofold expression level changes (308 upregulated and 332 downregulated), 163 genes with changes greater than threefold (65 upregulated and 98 downregulated), 111 genes with changes greater than fourfold (28 upregulated and 83 downregulated), 35 genes with changes greater than sixfold (11 upregulated and 24 downregulated), and 23 genes with changes greater than eightfold (eight upregulated and 15 downregulated) (Figure 2A). A graphical depiction of microarray gene expression results across 


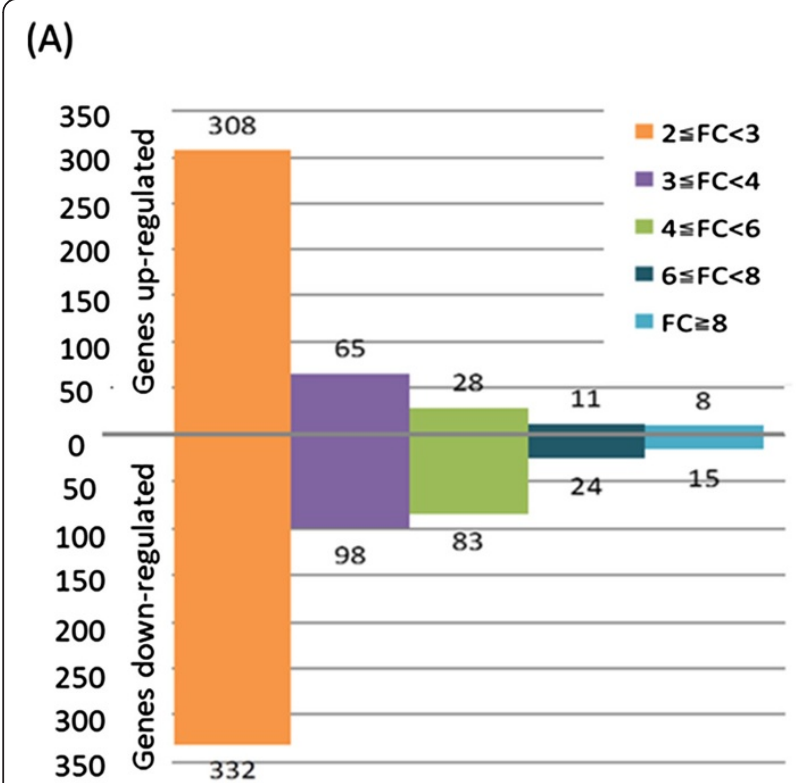

(C)

(B)
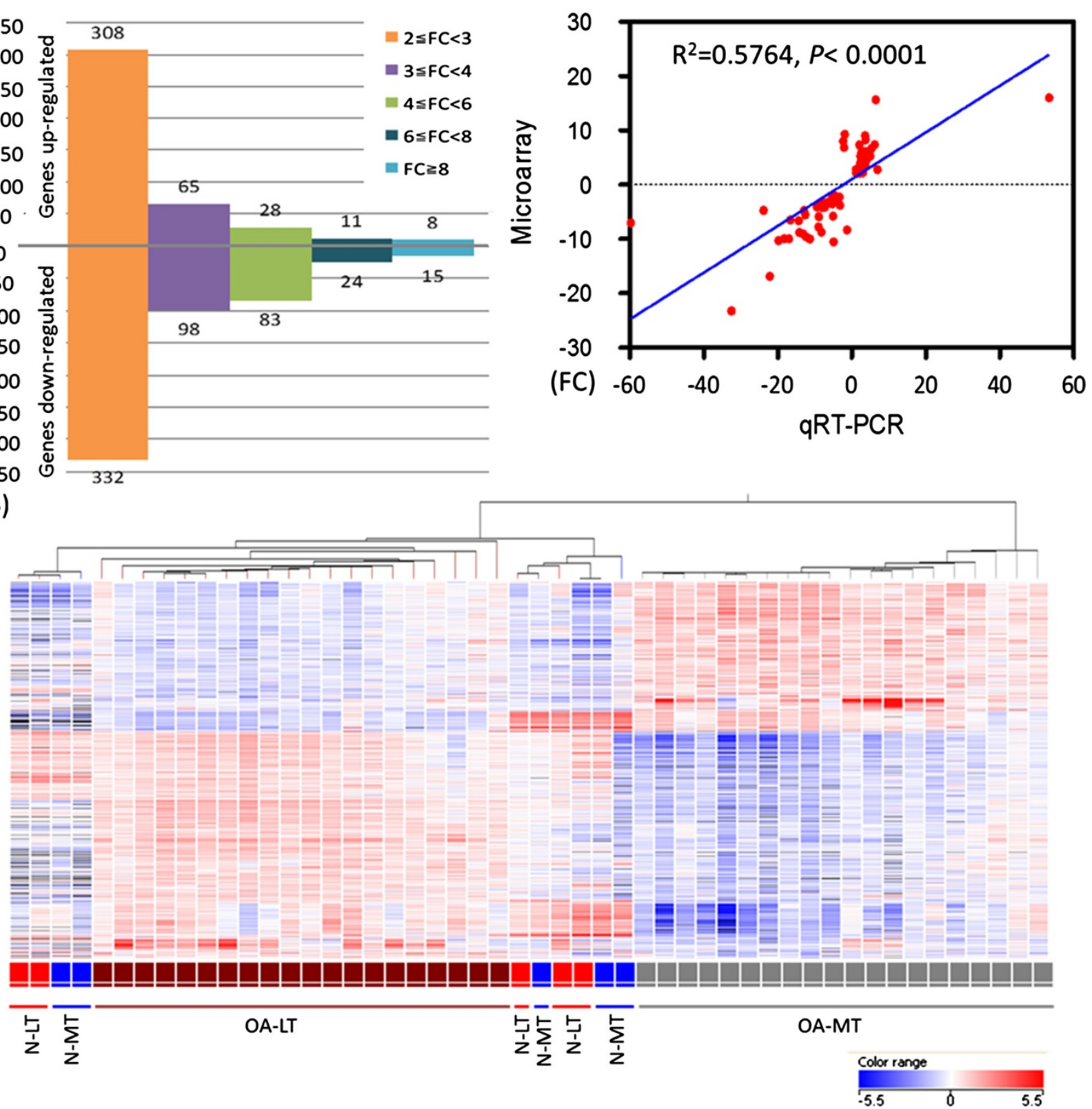

Figure 2 Microarray analyses of gene expression of osteoarthritis and non-osteoarthritis of subchondral bone and qRT-PCR validation in osteoarthritis knee subchondral bone. (A) Differentially expressed genes were classified based on their expression levels with a minimum of twofold, threefold, fourfold, sixfold, and eightfold changes. (B) Unsupervised hierarchical clustering of osteoarthritis (OA) and non-OA samples was performed for genes whose differential expression exceeded twofold (972 genes). Distances between samples were detected with a Euclidean algorithm and clustered with a centroid linkage method. The OA medial tibial (MT) samples (gray) were clustered together and clearly separated from the OA lateral tibial (LT) (brown) and non-OA samples. Non-OA LT samples (N-LT, red) and non-OA MT samples (N-MT, blue) were clustered together with OA LT samples. (C) Eighty-five genes (plus GAPDH) were selected for quantitative reverse-transcription polymerase chain reaction (qRT-PCR) validation on a separate group of nine OA subchondral bone specimens. The results of qRT-PCR were strongly consistent with those of microarray analysis $\left(R^{2}=0.5764, P<0.0001\right)$. FC, fold-change.

the entire extent of the tibial subchondral bone can be seen in Additional file 4.

Unsupervised hierarchical clustering was performed on the differentially expressed genes to identify the groups or clusters of genes based on similarities between the expression profiles of the samples. On the basis of gene expression profiles, OA LT and OA MT samples were grouped into two distinct clusters. However, the 
pattern for LT and MT samples from non-OA donors was mixed and did not cluster by sample site; the clustering of all non-OA samples was more akin to the OA LT samples than to the OA MT samples (Figure 2B).

\section{Quantitative reverse-transcription polymerase chain reaction validation}

Nine joint tissue samples were available for validation of microarray results; qRT-PCR analysis of LT and MT subchondral bone regions of these samples was performed on 86 selected genes with GAPDH as endogenous control. Based on the results of histological evaluation and microcomputed tomography scanning, the subchondral bone samples selected from the OA MT regions displayed characteristics of severe stages of OA; whereas the OA LT regions displayed characteristics of relatively normal joints. The mean expression pattern of the LT region was compared with the mean expression pattern of MT regions. By qRT-PCR, 77 of 85 genes showed a statistically significant difference between regions (Additional file 5). Expression by qRT-PCR matched the pattern (upregulation or downregulation) of expression observed by microarray, and the two datasets were strongly correlated $\left(R^{2}=0.5764, P<0001\right.$; Figure $\left.2 C\right)$. The most differentially upregulated genes in OA MT regions were IL11, POSTN, ASPN, COL6A3, TUBB3, COL3A1, OGN, DIO2, PPEF1, and TNFSF11. The most differentially downregulated genes in OA MT regions were $L E P, A P O B, A D H 1 B$, CCL8, TUSC5, KLB, NPR3, PLIN1, PCK1, and MYO16. All of these genes were validated by qRT-PCR except for $A D H 1 B$ and MYO16, because these two genes were not available on the microfluidic cards used for qRT-PCR (Table 2).

\section{Functional and pathway analysis}

IPA of gene expression profiles revealed 279 functional networks (canonical pathways) (Additional file 6) that were significantly associated with the differentially expressed genes. The predominant associated networks and biological functions were related to lipid metabolism and mineral metabolism (Additional files 7 and 8), connective tissue disorders, cellular growth and proliferation, connective tissue development and function (Additional file 9). Most of these pathways were associated with OA pathogenesis such as bone remodeling, adiposity, connective tissue, and cell activity and death (Table 3). Interestingly, on performing pathway analysis with the most upregulated and downregulated genes, a novel signaling network in OA was identified; this network was generally characterized by upregulation of bone mineralization or collagen-associated genes, such as COL3A1 (upregulated 7.33-fold, ranking sixth in the top upregulated gene list), COL6A3 (upregulated 8.27-fold, ranking fourth in the top upregulated gene list), $B M P 1, B M P 7, P O S T N$ (upregulated 15.65-fold, ranking second in the top upregulated gene list), WISP1, HTRA1, SOST, and ITGA11, and the downregulation of genes associated with cellular metabolism, proliferation or differentiation ( $N M B, L E P$ (downregulated 23.27-fold, ranking first in the top downregulated gene list), CHRDL2, GRB14, CIDEC, CIDEA, BTG2, PLAC8, and $P R D M 16)$. Additional differentially expressed genes in this network included ANGPTL1 (upregulated 2.16-fold), which is involved in angiogenesis. Both POSTN and LEP may affect the phosphorylation of $A K T$ signaling (Figure 3A).

\section{Validation of protein expression in subchondral bone specimens}

The regions of interest of subchondral bone samples ground for the gene expression study were about $5 \mathrm{~mm}$ deep under the calcified cartilage, which included the subchondral bone plate and subarticular spongiosa. These regions have a very heterogeneous complement of cells, including osteoblasts, osteoclasts, osteocytes and bone marrow cells. Although the specific cell types contributing to the changes in gene expression cannot easily be confirmed, all of the cell types in the subchondral bone would be expected to contribute to the subchondral bone gene expression profile. To obtain further independent validation of the microarray results, immunohistochemical staining was performed to investigate the protein expression of proteins Periostin and Leptin corresponding to genes POSTN and LEP in human OA and non-OA tissue sections from LT and MT regions. The highly expressed Periostin could be found in osteoblasts beneath the tidemark, in osteocytes, and in lining cells of the OA MT regions, but not OA LT regions (Figure 3B,C). Protein expression of Leptin was low in OA MT regions, but strong in OA LT regions (Figure 3D,E). By immunohistochemical analysis, the proteins Periostin and Leptin were not differentially expressed in the MT and LT regions of non-OA samples (Additional file 10).

\section{Discussion}

To the best of our knowledge, this represents the first comprehensive whole genome expression profiling of non-OA and OA subchondral bone. The validity of the microarray results was confirmed by qRT-PCR for a selected subset of genes. Based on analysis by IPA, we identified biological functions implicated in the pathogenesis of $\mathrm{OA}$ in bone. Many of the functional networks identified in this study were also associated with processes in connective tissue development and function.

In our previous study [15], we evaluated the expression of $61 \mathrm{OA}$ genes and correlated the expression patterns with the bone morphometric measurement (bone volume). The expression of a total of 45 of 61 genes in the subchondral bone was significantly correlated with 
Table 2 Top ten upregulated and downregulated genes comparing lateral tibial bone (below intact cartilage) with medial tibial bone (below lesioned cartilage)

\begin{tabular}{|c|c|c|c|c|c|c|}
\hline \multirow[b]{2}{*}{$\begin{array}{l}\text { Accession } \\
\text { number }\end{array}$} & \multirow[b]{2}{*}{$\begin{array}{c}\text { Gene } \\
\text { symbol }\end{array}$} & \multirow[b]{2}{*}{ Gene name } & \multirow[b]{2}{*}{$P$ value $^{\mathrm{a}}$} & \multicolumn{3}{|c|}{ Fold-change (MT vs. LT) } \\
\hline & & & & $\begin{array}{c}\text { OA } \\
\text { (microarray) }\end{array}$ & $\begin{array}{c}\text { Non-OA } \\
\text { (microarray) }\end{array}$ & $\begin{array}{c}\text { OA } \\
\text { (qRT-PCR) }\end{array}$ \\
\hline \multicolumn{7}{|c|}{ Genes with increased expression in medial tibial bone } \\
\hline NM_000641 & IL11 & Interleukin-11 & $1.32 \times 10^{-14}$ & 16.02 & 1.12 & $110.31^{*}$ \\
\hline NM_006475 & POSTN & Periostin, osteoblast specific factor & $1.48 \times 10^{-10}$ & 15.65 & 1.55 & $9.68^{*}$ \\
\hline NM_017680 & ASPN & Asporin & $2.82 \times 10^{-9}$ & 9.04 & 2.93 & $6.34^{*}$ \\
\hline NM_057165 & COL6A3 & Collagen, type VI, alpha 3 & $9.65 \times 10^{-10}$ & 8.27 & 1.33 & $5.11^{*}$ \\
\hline NM_006086 & TUBB3 & Tubulin, beta 3 & $6.98 \times 10^{-15}$ & 7.36 & 1.11 & $6.28^{*}$ \\
\hline NM_000090 & COL3A1 & Collagen, type III, alpha 1 & $5.33 \times 10^{-16}$ & 7.33 & 1.32 & $6.45^{*}$ \\
\hline NM_033014 & OGN & Osteoglycin & $7.24 \times 10^{-10}$ & 6.69 & 1.70 & $9.33^{*}$ \\
\hline NM_013989 & $\mathrm{D} / \mathrm{O} 2$ & Deiodinase, iodothyronine, type ॥ & $1.58 \times 10^{-11}$ & 6.20 & 1.31 & $5.74^{*}$ \\
\hline NM_006240 & PPEF1 & $\begin{array}{l}\text { Protein phosphatase, EF-hand calcium } \\
\text { binding domain } 1\end{array}$ & $5.31 \times 10^{-13}$ & 6.14 & 1.97 & $4.51^{*}$ \\
\hline NM_003701 & TNFSF11 & $\begin{array}{l}\text { Tumor necrosis factor (ligand) superfamily, } \\
\text { member } 11\end{array}$ & $1.21 \times 10^{-11}$ & 5.90 & 1.07 & $2.64^{*}$ \\
\hline \multicolumn{7}{|c|}{ Genes with decreased expression in medial tibial bone } \\
\hline NM_000230 & LEP & Leptin & $4.00 \times 10^{-10}$ & -23.27 & -2.05 & $-18.99 *$ \\
\hline NM_000384 & $A P O B$ & Apolipoprotein B & $3.31 \times 10^{-10}$ & -16.88 & -2.12 & $-10.20^{*}$ \\
\hline NM_000668 & $A D H 1 B$ & $\begin{array}{l}\text { Alcohol dehydrogenase 1B (class I), } \\
\text { beta polypeptide }\end{array}$ & $2.27 \times 10^{-7}$ & -12.23 & -1.95 & NA \\
\hline NM_005623 & CCL8 & Chemokine ( $\mathrm{C}-\mathrm{C}$ motif) ligand 8 & $8.17 \times 10^{-9}$ & -10.50 & -1.59 & $-6.00^{*}$ \\
\hline NM_172367 & TUSC5 & Tumor suppressor candidate 5 & $4.22 \times 10^{-10}$ & -10.25 & -1.89 & $-8.19^{*}$ \\
\hline NM_175737 & $K L B$ & Klotho beta & $2.24 \times 10^{-8}$ & -10.00 & -1.63 & $-6.09^{*}$ \\
\hline NM_001204375 & NPR3 & Natriuretic peptide receptor $C$ & $1.36 \times 10^{-10}$ & -9.95 & -1.38 & $-7.81^{*}$ \\
\hline NM_002666 & PLIN1 & Perilipin 1 & $2.98 \times 10^{-8}$ & -9.60 & -1.81 & $-5.88^{*}$ \\
\hline NM_002591 & PCK1 & Phosphoenolpyruvate carboxykinase 1 & $1.03 \times 10^{-9}$ & -9.01 & -1.87 & $-5.44^{*}$ \\
\hline NM_001198950 & MYO16 & Myosin XVI & $5.81 \times 10^{-7}$ & -8.84 & -1.93 & NA \\
\hline
\end{tabular}

LT, lateral tibial; MT, medial tibial; NA, not analyzed; OA, osteoarthritis. ${ }^{a}$ Adjusted $P$ value for microarray corrected for multiple testing by the Benjamini-Hochberg method. ${ }^{*} P<0.001$ for quantitative reverse-transcription polymerase chain reaction (qRT-PCR), comparing OA MT versus OA LT. $P$ values were determined by twotailed unpaired Student's $t$ test.

the alteration of bone structure including ADAMTS1, ASPN, BMP6, BMPER, CCL2, CCL8, COL5A1, COL6A3, COL7A1, COL16A1, FRZB, GDF10, MMP3, OGN, OMD, POSTN, PTGES, TNFSF11, WNT1 and others, including the ratio of OPG (also known as TNFRSF11B) to RANKL (also known as TNFSF11). RANKL is primarily produced from osteocytes, osteoblasts and/or marrow stromal cells and is a key factor for osteoclast differentiation and activation. RANKL binds to OPG, also known as osteoclastogenesis inhibitory factor, and interacts with the receptor (RANK) for RANKL, to modulate the level of osteoclast activity and regulate bone homeostasis in response to various endocrine, paracrine, cytokine and mechanical stresses $[17,18]$. As shown in Additional file 11, significant correlations were observed for OPG/RANKL and the bone parameters including percent bone volume, bone structure (structure model index), trabecular thickness and trabecular space. These data confirmed our hypothesis that investigations of subchondral bone gene expression changes could provide clues to the pathogenic mechanisms of OA and inform development of new diagnostic markers and therapeutic targets. We focused on the outer LT and central MT regions for the purposes of comprehensive microarray analyses in the current study. The characteristics of the central MT region generally included cartilage matrix loss, cyst formation within cartilage matrix, denuded sclerotic bone, and thickened subchondral bone plate, consistent with a bone sclerosis phenotype [19], whereas the outer LT region was relatively normal. Many of the most significantly regulated genes identified by this microarray study have documented roles in the pathogenesis of OA, arthritis or bone formation including seven of the top 10 upregulated genes (POSTN, ASPN, COL6A3, COL3A1, OGN, DIO2, TNFSF11) and two of the top 10 downregulated genes (LEP, APOB) [20-27]. Through IPA we could also identify the involvement of a 
Table 3 Genes representative of gene clusters associated with osteoarthritis

\begin{tabular}{|c|c|c|c|c|c|c|c|}
\hline \multirow{2}{*}{$\begin{array}{l}\text { Functional annotation } \\
\text { (number of genes, } P \text { value) }^{a}\end{array}$} & \multicolumn{3}{|c|}{ Representative genes } & \multirow[b]{2}{*}{$P$ value $^{\mathrm{b}}$} & \multicolumn{3}{|c|}{ Fold-change (MT vs. LT) } \\
\hline & $\begin{array}{l}\text { Accession } \\
\text { number }\end{array}$ & $\begin{array}{c}\text { Gene } \\
\text { symbol }\end{array}$ & Gene name & & $\begin{array}{c}\mathrm{OA} \\
\text { (microarray) }\end{array}$ & $\begin{array}{c}\text { Non-OA } \\
\text { (microarray) }\end{array}$ & $\begin{array}{c}\text { OA } \\
\text { (qRT-PCR) }\end{array}$ \\
\hline $\begin{array}{l}\text { Abnormal morphology } \\
\text { of bone }\end{array}$ & NM_004370 & COL12A1 & Collagen, type XII, alpha 1 & $6.55 \times 10^{-08}$ & 2.26 & 1.01 & 1.76 \\
\hline (42 genes, $P=1.5 \times 10^{-6}$ ) & NM_000594 & TNF & Tumor necrosis factor & $1.17 \times 10^{-08}$ & -3.17 & -1.45 & $-3.42^{*}$ \\
\hline $\begin{array}{l}\text { Abnormal morphology } \\
\text { of collagen fibrils }\end{array}$ & NM_000093 & COL5A1 & Collagen, type V, alpha 1 & $2.63 \times 10^{-08}$ & 2.90 & 1.35 & $6.40^{*}$ \\
\hline$\left(7\right.$ genes, $\left.P=1.68 \times 10^{-6}\right)$ & NM_000393 & COL5A2 & Collagen, type V, alpha 2 & $2.34 \times 10^{-10}$ & 3.12 & 1.40 & 3.18 \\
\hline \multirow[t]{2}{*}{ Adiposity (24 genes, $P<10^{-7}$ ) } & NM_003394 & WNT10B & $\begin{array}{l}\text { Wingless-type MMTV } \\
\text { integration site Family, } \\
\text { member } 10 \mathrm{~B}\end{array}$ & $9.76 \times 10^{-09}$ & 2.05 & -1.08 & NA \\
\hline & NM_001279 & CIDEA & $\begin{array}{l}\text { Cell death-inducing DFFA-like } \\
\text { effector a }\end{array}$ & $2.74 \times 10^{-09}$ & -6.26 & -1.71 & NA \\
\hline Bone mineral density & NM_025237 & SOST & Sclerostin & $2.59 \times 10^{-04}$ & 2.51 & 1.44 & $4.77^{*}$ \\
\hline (21 genes, $P=1.1 \times 10^{-5}$ ) & NM_005357 & LIPE & Lipase, hormone-sensitive & $4.46 \times 10^{-09}$ & -8.71 & -1.73 & $-4.21^{*}$ \\
\hline Angiogenesis & NM_004673 & ANGPTL 1 & Angiopoietin-like 1 & $2.55 \times 10^{-06}$ & 2.16 & 1.02 & NA \\
\hline (69 genes, $P<10^{-7}$ ) & NM_002982 & CCL2 & $\begin{array}{l}\text { Chemokine (C-C motif) } \\
\text { ligand } 2\end{array}$ & $3.08 \times 10^{-05}$ & -3.76 & -1.31 & $-4.87^{*}$ \\
\hline Osteoarthritis & NM_001851 & COL9A1 & Collagen, type IX, alpha 1 & $1.42 \times 10^{-06}$ & 2.19 & -1.05 & 2.35 \\
\hline (23 genes, $P<10^{-7}$ ) & NM_001463 & $F R Z B$ & Frizzled-related protein & $1.36 \times 10^{-10}$ & -3.69 & 1.38 & $-7.72^{*}$ \\
\hline Proliferation of cells & NM_001004439 & ITGA11 & integrin, alpha 11 & $4.05 \times 10^{-10}$ & 2.31 & -1.03 & NA \\
\hline \multirow[t]{3}{*}{ (303 genes, $P<10^{-7}$ ) } & NM_021077 & $N M B$ & Neuromedin B & $1.14 \times 10^{-06}$ & -3.00 & -1.36 & NA \\
\hline & NM_004490 & GRB14 & $\begin{array}{l}\text { Growth factor receptor- } \\
\text { bound protein } 14\end{array}$ & $3.38 \times 10^{-12}$ & -3.20 & -1.18 & NA \\
\hline & NM_006763 & BTG2 & BTG family, member 2 & $9.11 \times 10^{-08}$ & -3.67 & -1.31 & NA \\
\hline Quantity of connective tissue & NM_003474 & ADAM12 & $\begin{array}{l}\text { ADAM metallopeptidase } \\
\text { domain } 12\end{array}$ & $1.86 \times 10^{-10}$ & 2.30 & 1.10 & 1.93 \\
\hline (48 genes, $P<10^{-7}$ ) & NM_022094 & CIDEC & $\begin{array}{l}\text { Cell death-inducing DFFA-like } \\
\text { effector } c\end{array}$ & $2.08 \times 10^{-07}$ & -6.09 & -1.63 & NA \\
\hline Differentiation of cells & NM_054034 & FN1 & Fibronectin 1 & $1.44 \times 10^{-07}$ & 2.19 & 1.38 & $2.10^{*}$ \\
\hline (175 genes, $P<10^{-7}$ ) & NM_016619 & PLAC8 & Placenta-specific 8 & $1.01 \times 10^{-08}$ & -2.28 & 1.14 & NA \\
\hline Cell movement & NM_000094 & COL7A1 & Collagen, type VII, alpha 1 & $2.25 \times 10^{-09}$ & 2.28 & -1.00 & $3.81^{*}$ \\
\hline$\left(176\right.$ genes, $\left.P<10^{-7}\right)$ & NM_012193 & FZD4 & Frizzled family receptor 4 & $3.62 \times 10^{-10}$ & -3.02 & -1.37 & $4.93^{*}$ \\
\hline Necrosis & NM_080838 & WISP1 & $\begin{array}{l}\text { WNT1 inducible signaling } \\
\text { pathway protein } 1\end{array}$ & $3.79 \times 10^{-08}$ & 2.50 & 1.09 & NA \\
\hline (218 genes, $P<10^{-7}$ ) & NM_004797 & $A D I P O Q$ & $\begin{array}{l}\text { Adiponectin, } \mathrm{C} 1 \mathrm{Q} \text { and } \\
\text { collagen domain containing }\end{array}$ & $1.71 \times 10^{-07}$ & -6.63 & -1.69 & $10.51^{*}$ \\
\hline Mineralization of cells & NM_001719 & $B M P 7$ & $\begin{array}{l}\text { Bone morphogenetic } \\
\text { protein } 7\end{array}$ & $1.65 \times 10^{-09}$ & 2.50 & 1.34 & $2.26^{* *}$ \\
\hline$\left(14\right.$ genes, $\left.P=3 \times 10^{-6}\right)$ & NM_015424 & CHRDL2 & Chordin-like 2 & $1.40 \times 10^{-04}$ & -4.04 & 1.38 & $-15.71^{*}$ \\
\hline
\end{tabular}

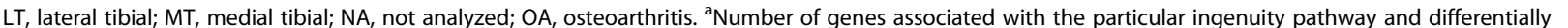
expressed in MT versus LT regions of OA samples by microarray analysis; $P$ value calculated by Fisher's exact test. ${ }^{b}$ Adjusted $P$ value for microarray corrected for multiple testing by the Benjamini-Hochberg method. ${ }^{*} P<0.001$ and ${ }^{*} P<0.05$ for quantitative reverse-transcription polymerase chain reaction (qRT-PCR); $P<0.05$ was considered significant comparing OA MT versus OA LT. $P$ values determined by two-tailed unpaired Student's $t$ test.

lipid metabolism protein network and a mineral metabolism protein network that involved 29 and 22 differentially expressed genes respectively; these networks included PLINE, LIPE, DGAT2, ADRB1, NPY1R, HCAR3 and $P 2 R R Y 14$ that changed -9.6 -fold, -8.7 -fold, -7.8 -fold, -6.2 -fold, -7.4 -fold, -5.1 -fold, and -5.1 -fold respectively comparing OA MT with OA LT (Additional files 7 and 8). These functional networks indicated that the bone cells in the OA MT regions were characterized by a condition of low energy production that included a low rate of mineral metabolism. These results are compatible with a low rate of bone remodeling in MT regions. 


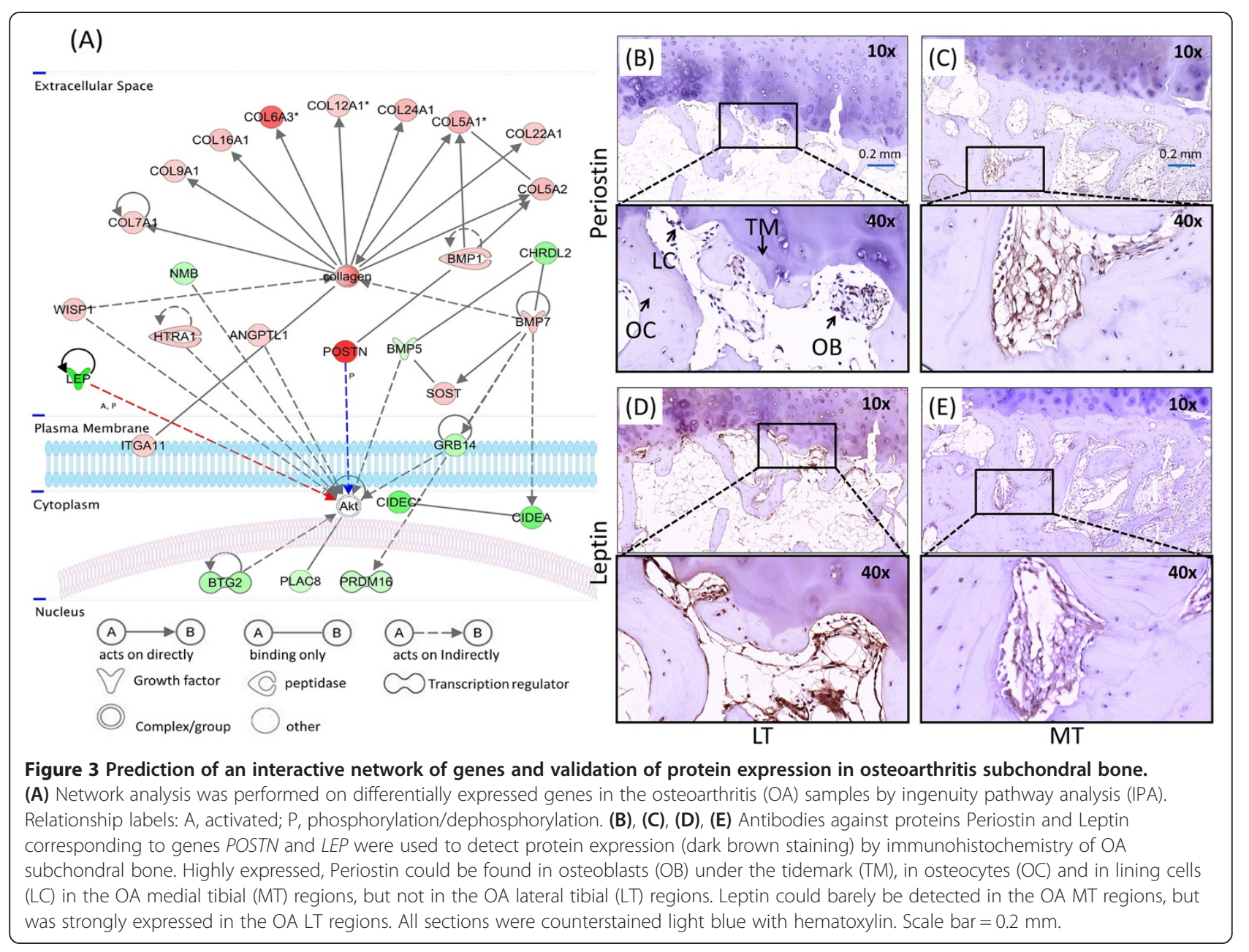

Increased bone remodeling is an important predictor of OA progression [8] and is characteristic of early stages of $\mathrm{OA}$ including resorption of bone and increased porosity in the subchondral bone region [28]. Given that OA MT regions of interest could be defined as representing late stages of OA, we were interested to determine whether OA LT regions of interest could be described as representing early stages of OA. The OARSI histopathological scores of all OA LT samples were $<6$, representing a relatively intact overlying cartilage matrix with minimal superficial fibrillation not too dissimilar from normal. However, in contrast to normal samples, the bone structural parameters of the OA LT regions had a lower bone volume, thinning of the subchondral bone plate, increased porosity in the subchondral region and reduced bone density (bone density data not shown) suggestive of early OA. Another indicator of early OA, as demonstrated by animal models of OA and in OA patients, is increased bone remodeling characterized by a decreased expression ratio of $O P G$ to $R A N K L$ [29,30]. In contrast to non-OA tissues, a reduced $O P G$ to $R A N K L$ ratio was identified in OA LT regions compared with non-OA LT regions (OPG lacked significant change, but RANKL was upregulated 1.63-fold, one- tailed unpaired Student $t$ test $P=0.048$ ), consistent with early OA. Thus, based on the bone parameters and $O P G$ to RANKL ratios, comparing LT with non-OA we concluded that the bone gene expression patterns of OA LT regions were consistent with an early-stage OA phenotype.

According to a hypothetical model of OA pathogenesis proposed by Burr and Gallant [6], joint loading increases bone remodeling in the early stage of $\mathrm{OA}$, and a bonecartilage crosstalk may occur via diffusion of small molecules or increased vascularization at the deep layers of cartilage that could interfere with the normal collagen network. These changes would lead to a loss of aggrecan, increasing inflammation, and result in bone sclerosis at late stages of OA. Several of the genes identified in this study fit nicely into this hypothetical model. For instance, upregulation of SOST was identified (2.51-fold); SOST is an inhibitor of Wnt signaling that responds to mechanical loading in the proximal rat tibia and is associated with bone mass changes [31]. In addition, several genes relevant to cell differentiation and activity of osteoblasts, osteoclasts and osteocytes were identified in both a mouse load-regulated gene expression model [32] and in this 
human study; examples included ASPN (regulator of osteoblast collagen mineralization), WNT5A (agonist of WNT signaling pathway in osteoblasts and osteocytes) and VCAN (regulating transforming growth factor beta expression in osteoclasts). These findings suggest that mechanical loading plays an important role in structural changes of human subchondral bone, initiates bone remodeling, and contributes to the pathogenesis of OA. Moreover, our results could provide a possible novel molecular mechanism to explain changes in bone remodeling during OA development based on an AKT-regulated network. AKT is a serine/threonine-specific protein kinase that plays a pivotal role in many cellular processes such as metabolism, apoptosis, cell proliferation, transcription, cell migration and extracellular matrix alternation [33]. In an early-stage of OA, overexpressed LEP may increase phosphorylation of AKT [34], which will trigger the downstream signaling pathway to increase bone remodeling. In a late stage of OA, upregulated POSTN may inhibit phosphorylation of AKT [35] and will decrease the cellular metabolism, cell proliferation, and differentiation; the rate of bone remodeling would thereby decline.

One of the limitations of this study was that the subchondral bone samples were obtained from OA joints at end-stage disease due to the difficulty of obtaining joints with early stages of OA from humans. However, comparing our results with the gene expression profiles of subchondral bone in an early OA experimental mouse model [12], their reported fold-changes in SB gene expression of ASPN, CCL2, COL3A1, COL5A1, POSTN and TNFSF11 at the initial stages of OA were similar to those of our study. This result strongly supports the credibility of this study. Another limitation was the difficulty distinguishing whether differentially expressed genes reflected OA progression in cartilage, differences in innate bone structure, or were driven by changes in mechanical loading. In our currently study, significant correlation between overlying cartilage integrity and underlying subchondral bone structural parameters could be identified (Additional file 12), suggesting that differential gene expression across the subchondral bone is due, at least in part, to differences in cartilage integrity of the LT and MT regions in OA. Microarray analysis of additional non-OA control samples could help to exclude differentially expressed genes driven by innate bone structure. However, to exclude genes driven by mechanical loading, microarray analysis of lateral compartment dominant $\mathrm{OA}$ is necessary.

The site of initiation of OA, at bone or cartilage, has been disputed for decades. Numerous clinical and experimental studies have confirmed that increased bone volume and changes in bone quality of the tibial subchondral bone of the knee are related to loss of cartilage integrity [7,8,36-38]. Goldring and Goldring have pointed out that subchondral bone responds more rapidly to adverse loading and events than cartilage [5]. Moreover, the integrity of subchondral bone predicts later worsening of radiographic osteoarthritis progression [8]. As summarized by Martel-Pelletier and Pelletier, an abnormal rate of bone remodeling, incomplete bone mineralization and increased osteoid collagen matrix will result in hypomineralization of subchondral bone and deterioration of cartilage as manifested by knee OA progression [39]. These data would suggest that drugs designed to target both the bone and cartilage compartments to optimize bone remodeling rates and inhibit cartilage loss in the early stages of OA could inhibit progression of disease. The recent success of strontium ranelate as a disease-modifying agent for OA provides the first clear evidence that this hypothesis has been confirmed [40]. Some other bone-targeting agents have been assessed as OA therapeutics, including bisphosphonates [41,42], calcitonin [43] and vitamin D [44]. These bone-acting agents are unlikely to be effective in late stages of $\mathrm{OA}$, when remodeling is already suppressed. The development of diagnostic biomarkers to detect progressive changes in early stages of $\mathrm{OA}$ is therefore important. The genes highly regulated in early stages of OA in our microarray data could provide potential diagnostic biomarkers or therapeutic targets for OA.

\section{Conclusion}

Our microarray results were obtained by examining early and late stages of OA and non-OA samples. Most of the differentially expressed genes in bone are involved in cartilage and bone development, OA pathogenesis and bone remodeling in the early and late stages of OA. This direct assessment of gene expression profiling in OA subchondral bone provides a comprehensive understanding of disease pathology that could further the development of new OA biomarkers and therapeutic strategies.

\section{Additional files}

Additional file 1: Shows sites of analysis and regions for RNA
isolation.
Additional file 2: Shows self-organizing maps analysis (SOM) of the
differentially expressed genes across the entire tibial plateau.
Additional file 3: Presents characteristics of the paired and
unpaired OA samples used for microarray analysis.
Additional file 4: Presents a list of differentially expressed genes.
Additional file 5: Presents gene validation by qRT-PCR.
Additional file 6: Presents a list of canonical pathways.
Additional file 7: Shows a potential network of lipid metabolism in OA.
Additional file 8: Shows a potential network of mineral metabolism in OA.
Additional file 9: Presents a list of dominant functional networks.
Additional file 10: Shows validation of protein expression in
human non-OA knee samples.
Additional file 11: Presents correlation of OPG/RANKL and
structural parameters of the subchondral bone.
Additional file 12: Presents correlations between cartilage integrity
and the bone parameters.




\begin{abstract}
Abbreviations
IPA: Ingenuity pathway analysis; LT: Lateral tibial; MT: Medial tibial; OA: Osteoarthritis; OARSI: Histological scoring system to grade severity of osteoarthritis; qRT-PCR: Quantitative reverse-transcription polymerase chain reaction.
\end{abstract}

\section{Competing interests}

The authors declare that they have no competing interests.

\section{Authors' contributions}

C-HC carried out the RNA isolation, micro-computed tomography, GRT-PCR, microarray data analysis, statistical analysis, histology and immunohistochemistry experiments, and drafted the manuscript. C-CW, C-HL, H-PC, I-WS, J-HC, L-SL and S-YK participated in RNA isolation and sample collection. J-YW, Y-TC, VBK, and MTML conceived of the study, participated in its design and coordination, and helped to draft the manuscript. MTML had full access to all of the data in the study and takes responsibility for the integrity of the data and the accuracy of the data analysis. All authors read and approved the final version to be published.

\section{Acknowledgements}

This study was supported by the Academia Sinica Genomic Medicine Multicenter Study (40-05-GMM), the National Research Program for Genomic Medicine, National Science Council, Taiwan (Translational Resource Center for Genomic Medicine: NSC101-2325-B-001-035, National Center for Genome Medicine: NSC101-2319-B-001-001, and MTML: NSC101-2320-B-001-020-MY3), NIH/NIA Claude D. Pepper OAIC 5P30 AG028716 and P01 AR50245 (VBK), and an OARSI scholarship (to $\mathrm{C}-\mathrm{HC}$ ).

The authors would like to thank the Translational Resource Center for Genomic Medicine of the National Research Program for Biopharmaceuticals, for the support in project management, and the Taiwan Mouse Clinic, which is funded by the National Research Program for Biopharmaceuticals at the National Science Council of Taiwan for technical support in micro-computed tomography experiments.

\section{Author details}

${ }^{1}$ Institute of Biomedical Sciences, Academia Sinica, 128, Sec. 2, Academia Road, Nankang District, Taipei 11529, Taiwan. ${ }^{2}$ National Center for Genome Medicine, Institute of Biomedical Sciences, Academia Sinica, 128, Sec. 2, Academia Road, Nankang District, Taipei 11529, Taiwan. ${ }^{3}$ Department of Pathology, Duke University School of Medicine, DUMC 3712, Durham, NC 27710, USA. ${ }^{4}$ Department of Orthopaedic Surgery, Tri-Service General Hospital, National Defense Medical Center, 325, Sec. 2, Cheng-gung Rd., Neihu Dist., Taipei 11472, Taiwan. ${ }^{5}$ Graduate Institute of Life Sciences, Tri-Service General Hospital, National Defense Medical Center, 114 No.161, Sec. 6, Minquan E. Rd., Neihu Dist., Taipei 114, Taiwan. ${ }^{6}$ Translational Resource Center for Genomic Medicine, Institute of Biomedical Sciences, Academia Sinica, 128, Sec. 2, Academia Rd., Nankang District, Taipei 11529, Taiwan. ${ }^{7}$ Department of Orthopedics, School of Medicine, College of Medicine, Taipei Medical University, 250 Wu-Hsing Street, Taipei 110, Taiwan. ${ }^{8}$ Department of Orthopedics, Taipei Medical University Hospital, 250 Wu-Hsing Street, Taipei 110, Taiwan. ${ }^{9}$ Department of Pediatrics, Duke University School of Medicine, DUMC 3352, Durham, NC 27710, USA. ${ }^{10}$ Department of Medicine, Duke University School of Medicine, 2301 Erwin Road, Room 1102, Durham, NC 27710, USA. ${ }^{11}$ Graduate Institute of Chinese Medical Science, China Medical University, No.91 Hsueh-Shih Road, Taichung 40402, Taiwan. ${ }^{12}$ Laboratory for International Alliance on Genomic Research, RIKEN Center for Integrative Medical Sciences, 1-7-22 Suehiro-cho, Tsurumi-ku, Yokohama, Kanagawa 230-0045, Japan.

Received: 22 February 2013 Accepted: 1 November 2013 Published: 15 November 2013

\section{References}

1. Lawrence RC, Felson DT, Helmick CG, Arnold LM, Choi H, Deyo RA, Gabriel S, Hirsch R, Hochberg MC, Hunder GG, Jordan JM, Katz JN, Kremers HM, Wolfe F: National Arthritis Data Workgroup: Estimates of the prevalence of arthritis and other rheumatic conditions in the United States. Part II. Arthritis Rheum 2008, 58:26-35

2. Fransen $M$, Bridgett $L$, March L, Hoy D, Penserga E, Brooks P: The epidemiology of osteoarthritis in Asia. Int J Rheum Dis 2011, 14:113-121.
3. Grynpas MD, Alpert B, Katz I, Lieberman I, Pritzker KP: Subchondral bone in osteoarthritis. Calcif Tissue Int 1991, 49:20-26.

4. Brandt KD, Radin EL, Dieppe PA, van de Putte L: Yet more evidence that osteoarthritis is not a cartilage disease. Ann Rheum Dis 2006, 65:1261-1264.

5. Goldring MB, Goldring SR: Articular cartilage and subchondral bone in the pathogenesis of osteoarthritis. Ann N Y Acad Sci 2010, 1192:230-237.

6. Burr DB, Gallant MA: Bone remodelling in osteoarthritis. Nat Rev Rheumatol 2012, 8:665-673.

7. Ding C, Cicuttini F, Jones G: Tibial subchondral bone size and knee cartilage defects: relevance to knee osteoarthritis. Osteoarthritis Cartilage 2007, 15:479-486.

8. Kraus VB, Feng S, Wang S, White S, Ainslie M, Brett A, Holmes A, Charles HC: Trabecular morphometry by fractal signature analysis is a novel marker of osteoarthritis progression. Arthritis Rheum 2009, 60:3711-3722.

9. Huebner JL, Hanes MA, Beekman B, TeKoppele JM, Kraus VB: A comparative analysis of bone and cartilage metabolism in two strains of guinea-pig with varying degrees of naturally occurring osteoarthritis. Osteoarthritis Cartilage 2002, 10:758-767.

10. Westacott Cl, Webb GR, Warnock MG, Sims JV, Elson CJ: Alteration of cartilage metabolism by cells from osteoarthritic bone. Arthritis Rheum 1997, 40:1282-1291.

11. Sanchez C, Deberg MA, Piccardi N, Msika P, Reginster JY, Henrotin YE: Osteoblasts from the sclerotic subchondral bone downregulate aggrecan but upregulate metalloproteinases expression by chondrocytes. This effect is mimicked by interleukin- $6,-1 \beta$ and oncostatin $M$ pre-treated non-sclerotic osteoblasts. Osteoarthritis Cartilage 2005, 13:979-987.

12. Zhang R, Fang H, Chen Y, Shen J, Lu H, Zeng C, Ren J, Zeng H, Li Z, Chen S, Cai D, Zhao Q: Gene expression analyses of subchondral bone in early experimental osteoarthritis by microarray. PLoS One 2012, 7:e32356.

13. Hopwood B, Tsykin A, Findlay DM, Fazzalari NL: Microarray gene expression profiling of osteoarthritic bone suggests altered bone remodelling. WNT and transforming growth factor-beta/bone morphogenic protein signalling. Arthritis Res Ther 2007, 9:R100.

14. Hopwood B, Gronthos S, Kuliwaba JS, Robey PG, Findlay DM, Fazzalari NL: Identification of differentially expressed genes between osteoarthritic and normal trabecular bone from the intertrochanteric region of the proximal femur using cDNA microarray analysis. Bone 2005, 36:635-644.

15. Chou CH, Lee CH, Lu LS, Song IW, Chuang HP, Kuo SY, Wu JY, Chen YT, Kraus VB, Wu CC, Lee MT: Direct assessment of articular cartilage and underlying subchondral bone reveals a progressive gene expression change in human osteoarthritic knees. Osteoarthritis Cartilage 2013, 21:450-461.

16. Altman R, Asch E, Bloch D, Bole G, Borenstein D, Brandt K, Christy W, Cooke TD, Greenwald R, Hochberg M, et al: Development of criteria for the classification and reporting of osteoarthritis. Classification of osteoarthritis of the knee. Diagnostic and Therapeutic Criteria Committee of the American Rheumatism Association. Arthritis Rheum 1986, 29:1039-1049.

17. Nakashima T, Hayashi M, Fukunaga T, Kurata K, Oh-Hora M, Feng JQ, Bonewald LF, Kodama T, Wutz A, Wagner EF, Penninger JM, Takayanagi H: Evidence for osteocyte regulation of bone homeostasis through RANKL expression. Nat Med 2011, 17:1231-1234.

18. Lacey DL, Boyle WJ, Simonet WS, Kostenuik PJ, Dougall WC, Sullivan JK, San Martin J, Dansey R: Bench to bedside: elucidation of the OPG-RANKRANKL pathway and the development of denosumab. Nat Rev Drug Discov 2012, 11:401-419.

19. Karsdal MA, Leeming DJ, Dam EB, Henriksen K, Alexandersen P, Pastoureau P, Altman RD, Christiansen C: Should subchondral bone turnover be targeted when treating osteoarthritis? Osteoarthritis Cartilage 2008, 16:638-646.

20. Loeser RF, Olex AL, McNulty MA, Carlson CS, Callahan MF, Ferguson CM, Chou J, Leng $X$, Fetrow JS: Microarray analysis reveals age-related differences in gene expression during the development of osteoarthritis in mice. Arthritis Rheum 2012, 64:705-717.

21. Fukui N, Miyamoto $Y$, Nakajima M, Ikeda Y, Hikita A, Furukawa H, Mitomi H, Tanaka N, Katsuragawa Y, Yamamoto S, Sawabe M, Juji T, Mori T, Suzuki R, Ikegawa S: Zonal gene expression of chondrocytes in osteoarthritic cartilage. Arthritis Rheum 2008, 58:3843-3853.

22. Meulenbelt I, Min JL, Bos S, Riyazi N, Houwing-Duistermaat JJ, van der Wijk $H J$, Kroon HM, Nakajima M, Ikegawa S, Uitterlinden AG, van Meurs JB, van der Deure WM, Visser TJ, Seymour AB, Lakenberg N, van der Breggen R, Kremer D, van Duijn CM, Kloppenburg M, Loughlin J, Slagboom PE: Identification of $\mathrm{DIO} 2$ as a new susceptibility locus for symptomatic osteoarthritis. Hum Mol Genet 2008, 17:1867-1875. 
23. Wildey GM, Billetz AC, Matyas JR, Adams ME, McDevitt CA: Absolute concentrations of mRNA for type I and type VI collagen in the canine meniscus in normal and ACL-deficient knee joints obtained by RNase protection assay. J Orthop Res 2001, 19:650-658.

24. Tanaka K, Matsumoto E, Higashimaki Y, Katagiri T, Sugimoto T, Seino S, Kaji $\mathrm{H}$ : Role of osteoglycin in the linkage between muscle and bone. J Biol Chem 2012, 287:11616-11628.

25. Qin J, Shi D, Dai J, Zhu L, Tsezou A, Jiang Q: Association of the leptin gene with knee osteoarthritis susceptibility in a Han Chinese population: a case-control study. J Hum Genet 2010, 55:704-706.

26. Sanchez-Enriquez S, Torres-Carrillo NM, Vazquez-Del Mercado M, Salgado-Goytia L, Rangel-Villalobos H, Munoz-Valle JF: Increase levels of apo-A1 and apo B are associated in knee osteoarthritis: lack of association with VEGF-460 T/C and +405 C/G polymorphisms. Rheumatol Int 2008, 29:63-68.

27. Haringman JJ, Smeets TJ, Reinders-Blankert P, Tak PP: Chemokine and chemokine receptor expression in paired peripheral blood mononuclear cells and synovial tissue of patients with rheumatoid arthritis, osteoarthritis, and reactive arthritis. Ann Rheum Dis 2006, 65:294-300.

28. Pastoureau PC, Chomel AC, Bonnet J: Evidence of early subchondral bone changes in the meniscectomized guinea pig. A densitometric study using dual-energy X-ray absorptiometry subregional analysis. Osteoarthritis Cartilage 1999, 7:466-473.

29. Bellido M, Lugo L, Roman-Blas JA, Castaneda S, Caeiro JR, Dapia S, Calvo E, Largo R, Herrero-Beaumont G: Subchondral bone microstructural damage by increased remodelling aggravates experimental osteoarthritis preceded by osteoporosis. Arthritis Res Ther 2010, 12:R152.

30. Tat SK, Pelletier JP, Lajeunesse D, Fahmi H, Duval N, Martel-Pelletier J: Differential modulation of RANKL isoforms by human osteoarthritic subchondral bone osteoblasts: influence of osteotropic factors. Bone 2008, 43:284-291.

31. Macias BR, Aspenberg P, Agholme F: Paradoxical Sost gene expression response to mechanical unloading in metaphyseal bone. Bone 2013, 53:515-519.

32. Wasserman E, Webster D, Kuhn G, Attar-Namdar M, Muller R, Bab I: Differential load-regulated global gene expression in mouse trabecular osteocytes. Bone 2013, 53:14-23.

33. Ikegami D, Akiyama H, Suzuki A, Nakamura T, Nakano T, Yoshikawa $H$, Tsumaki N: Sox9 sustains chondrocyte survival and hypertrophy in part through Pik3ca-Akt pathways. Development 2011, 138:1507-1519.

34. Yang $R$, Barouch LA: Leptin signaling and obesity: cardiovascular consequences. Circ Res 2007, 101:545-559.

35. Kim CJ, Sakamoto K, Tambe Y, Inoue H: Opposite regulation of epithelial-to-mesenchymal transition and cell invasiveness by periostin between prostate and bladder cancer cells. Int J Oncol 2011, 38:1759-1766.

36. Muraoka T, Hagino H, Okano T, Enokida M, Teshima R: Role of subchondral bone in osteoarthritis development: a comparative study of two strains of guinea pigs with and without spontaneously occurring osteoarthritis. Arthritis Rheum 2007, 56:3366-3374.

37. Cox LG, van Rietbergen B, van Donkelaar CC, Ito K: Bone structural changes in osteoarthritis as a result of mechanoregulated bone adaptation: a modeling approach. Osteoarthritis Cartilage 2011, 19:676-682

38. Weinans H, Siebelt M, Agricola R, Botter SM, Piscaer TM, Waarsing JH: Pathophysiology of peri-articular bone changes in osteoarthritis. Bone 2012, 51:190-196.

39. Martel-Pelletier J, Pelletier JP: Is osteoarthritis a disease involving only cartilage or other articular tissues? Eklem hastaliklari ve cerrahisi 2010, 21:2-14.

40. Reginster JY, Badurski J, Bellamy N, Bensen W, Chapurlat R, Chevalier X, Christiansen C, Genant H, Navarro F, Nasonov E, Sambrook PN, Spector TD, Cooper C: Efficacy and safety of strontium ranelate in the treatment of knee osteoarthritis: results of a double-blind, randomised placebo-controlled trial. Ann Rheum Dis 2012, 72:179-186.

41. Moreau M, Rialland P, Pelletier JP, Martel-Pelletier J, Lajeunesse D, Boileau C, Caron J, Frank D, Lussier B, del Castillo JR, Beauchamp G, Gauvin D, Bertaim T, Thibaud D, Troncy E: Tiludronate treatment improves structural changes and symptoms of osteoarthritis in the canine anterior cruciate ligament model. Arthritis Res Ther 2011, 13:R98.
42. Walsh DA, Chapman V: Bisphosphonates for osteoarthritis. Arthritis Res Ther 2011, 13:128.

43. Castaneda S, Roman-Blas JA, Largo R, Herrero-Beaumont G: Subchondral bone as a key target for osteoarthritis treatment. Biochem Pharmacol 2012, 83:315-323.

44. Arabelovic S, McAlindon TE: Considerations in the treatment of early osteoarthritis. Curr Rheumatol Rep 2005, 7:29-35.

doi:10.1186/ar4380

Cite this article as: Chou et al:: Genome-wide expression profiles of subchondral bone in osteoarthritis. Arthritis Research \& Therapy 2013 15:R190.

\section{Submit your next manuscript to BioMed Central and take full advantage of:}

- Convenient online submission

- Thorough peer review

- No space constraints or color figure charges

- Immediate publication on acceptance

- Inclusion in PubMed, CAS, Scopus and Google Scholar

- Research which is freely available for redistribution 